\title{
Model Reduction of LFT Systems
}

\author{
Weizheng Wang, John Doyle and Carolyn Beck \\ Electrical Engineering, M/S 116-81 \\ California Institute of Technology \\ Pasadena, CA 91125
}

\author{
Keith Glover \\ Department of Electrical Engineering \\ University of Cambridge \\ Cambridge, CB2 1PZ, U.K.
}

\begin{abstract}
The notion of balanced realizations and balanced truncation model reduction, including guaranteed error bounds, is extended to general $\mathcal{Q}$-stable Linear Fractional Transformations (LFTs). Since both multidimensional and uncertain systems are naturally represented using LFTs, this can be interpreted either as doing state order reduction for multi-dimensional (MD) systems, or as uncertainty simplification in the case of uncertain systems. The role of Lyapunov equations in the 1-D theory is replaced by Linear Matrix Inequalities (LMIs). All proofs are given in detail as they are very short and in fact, greatly simplify even the standard 1-d case. This illustrates the power and generality of the LFT/ $\mu / \mathrm{LMI}$ machinery used throughout this paper.
\end{abstract}

\section{Introduction}

This paper applies the machinery of Linear Fractional Transforma tions (LFTs), the Structured Singular Value $(\mu)$, and Linear Matrix Inequalities (LMIs) reviewed in [6] to the problem of model reduction. Consider the system shown in Figure 1.

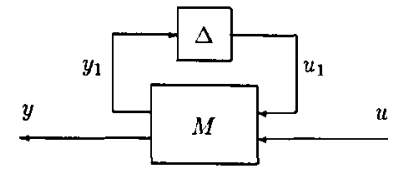

Figure 1: MD System

Let the frequency/uncertainty structure ([6]) $\Delta \in \Delta$ be

$$
\begin{gathered}
\Delta=\left\{\operatorname{diag}\left[\delta_{1} I_{q_{1}}, \ldots, \delta_{r} I_{q_{r}}\right]: \delta_{i} \in \mathrm{C}\right\} \\
\mathbf{B} \boldsymbol{\Delta}=\{\Delta \in \Delta: \bar{\sigma}(\Delta) \leq 1\}
\end{gathered}
$$

While we will only consider scalar times identity blocks in this paper, the generalization of the results to include full blocks is straightforward, though notationally cumbersome. For this paper, we will require the system to be $\mathcal{Q}$ stable ([6]), which will be reviewed below.

This system may be interpreted in a number of ways, although the results are independent of these interpretations. The most obvious is to view the $\delta_{i}$ as different transform variables in a multidimensional (MD) system ([10]) with transfer function $\mathcal{F}_{u}(M, \Delta)$. In this case, model reduction means state order reduction, similar to the standard 1-d case. We would like to find reduced order models which match the full order model well at all values of the $\delta_{i}$ on the polydisc $\left|\delta_{i}\right|=1, \forall i$. The results in this paper are directly relevant to this interpretation, as we obtain reduced order models $\mathcal{F}_{u}(\hat{M}, \hat{\Delta})$ with guaranteed bounds of the form

$$
\left\|\mathcal{F}_{u}(M, \Delta)-\mathcal{F}_{u}(\hat{M}, \hat{\Delta})\right\|_{\infty} \leq 2 \sum_{i=1}^{r} \sum_{i=k_{i}+1}^{t_{i}} \sigma_{i j}
$$

Although the exact meaning of this notation will require some development, we may note that in the $1-d$ case (3) reduces to the standard bound for balanced truncation ([7], [8], [9]).

As in [6], we may also view one of the $\delta_{i}$, say $\delta_{1}$ as the frequency variable $\left(\delta_{1}=1 / z\right)$ in an uncertain discrete-time system. The remaining $\delta_{i}$ are then viewed as norm-bounded perturbations. Model reduction in this context is aimed at simplifying the uncertainty description as well as reducing the state dimension and is a more subtle issue. While (3) has a natural interpretation in this case similar to the MD case, we might prefer model reduction of a much different nature. For example, there might be instances where we would be happy with reduced models which "cover" our uncertainty, rather than matching it, as is implied by (3). We might seek methods which aggregate several uncertainties into a simpler but larger uncertainty, and so on. These issues are not addressed specifically in this paper, but it is hoped that the LFT machinery used herein will lead to additional methods.

For one-dimensional systems with no uncertainty, there are a number of model reduction methods and associated error bounds. Examples include the balanced model reduction method and its additive $H_{\infty}$ norm error bound ([12], [7], [8], [9]); the optimal Hankel norm model reduction method and its Hankel norm error bound ([8]); and the balanced stochastic truncation model reduction method and its relative $H_{\infty}$ norm error bound ([5], [15]). Previously, there have been no algorithms and associated error bounds available for MD system model reduction. A model reduction algorithm and error bound for 2-dimensional systems have been developed in [14]. Unfortunately, the analysis techniques presented in [14] are applicable only to a special case of 2-dimensional systems. Using the machinery associated with LFTs, we extend the method of balanced model reduction to MD systems.

In Section 2, we review balanced realizations and the error bounds associated with model reduction for the 1-D case. The proofs are elementary and directly generalize to the MD case. In Section 3, the MD case is considered. Balanced realizations, gramians, and quadratic stability ( $\mathcal{Q}$-stability) are defined for MD systems. The proofs for the 1$D$ case are then extended in a natural way to the MD case. While only the discrete-time case is considered here, the continuous case follows immediately by simply transforming the results using the LFT for disk to half-plane.

\section{1-D systems}

In this section, we discuss balanced model reduction in the 1-D case, using machinary that directly generalizes to the M-D case. A new proof for the $H_{\infty}$ norm error bound is presented.

\subsection{Review of Balanced Realizations}

Suppose $G=\left[\begin{array}{c|c}A & B \\ \hline C & D\end{array}\right]$ is a discrete time system with $A$ stable. Let $Y$ and $X$ be the controllability and observability gramians, respectively, which satisfy

$$
\begin{aligned}
& A Y A^{*}-Y+B B^{*}=0 \\
& A^{*} X A-X+C^{*} C=0 .
\end{aligned}
$$


with $Y \geq 0, X \geq 0$. Standard results from the theory of Lyapunov equations state that the pair $(A, B)$ is controllable if and only if $Y>0$, and $(C, A)$ is observable if and only if $X>0$.

Suppose the state is transformed by a nonsingular $T$ to $\hat{x}=T x$ to yield the realization

$$
G=\left[\begin{array}{c|c}
\hat{A} & \hat{B} \\
\hline \hat{C} & \hat{D}
\end{array}\right]=\left[\begin{array}{c|c}
T A T^{-1} & T B \\
\hline C T^{-1} & D
\end{array}\right]
$$

Then the gramians are transformed to $\hat{Y}=T Y T^{*}$ and $\hat{X}=$ $\left(T^{-1}\right)^{*} X T^{-1}$. Consider the similarity transformation $T$ which gives the eigenvector decomposition

$$
Y X=T \Lambda T^{-1}, \Lambda=\operatorname{diag}\left(\lambda_{1}, \ldots, \lambda_{n}\right)
$$

Then the columns of $T$ are eigenvectors of $Y X$ corresponding to the eigenvalues $\left\{\lambda_{i}\right\}$. Since $Y \geq 0$ and $X \geq 0$, it can be shown that $Y X$ has a real diagonal Jordan form and that $\Lambda \geq 0$.

Although the eigenvectors are not unique, in the case of a minimal realization they can always be chosen such that

$$
\hat{Y}=T Y T^{*}=\Sigma \quad \text { and } \quad \hat{X}=\left(T^{-1}\right)^{*} X T^{-1}=\Sigma,
$$

where $\Sigma=\operatorname{diag}\left(\sigma_{1}, \sigma_{2}, \ldots, \sigma_{n}\right) \geq 0$ and $\Sigma^{2}=\Lambda$. This new realization with controllability and observability gramians $\hat{Y}=\hat{X}=\Sigma$ is referred to as a balanced realization.

More generally, if the realization of a system is not minimal, then there is a transformation such that the controllability and observability gramians are diagonal and the controllable and observable subsystem is balanced. The following theorem is standard, so the proof is omitted.

Theorem 1 For any stable system $G=\left[\begin{array}{c|c}A & B \\ \hline C & D\end{array}\right]$ there exists $T$ such that $G=\left[\begin{array}{c|c}T A T^{-1} & T B \\ \hline C T^{-1} & D\end{array}\right]$ has controllability and observability grami-
ans given by

$$
Y=\left[\begin{array}{cccc}
\Sigma_{1} & & & \\
& \Sigma_{2} & & \\
& & 0 & \\
& & & 0
\end{array}\right] \text { and } X=\left[\begin{array}{cccc}
\Sigma_{1} & & & \\
& 0 & & \\
& & \Sigma_{3} & \\
& & & 0
\end{array}\right]
$$

respectively, with $\Sigma_{1}, \Sigma_{2}, \Sigma_{3}$ diagonal and positive definite.

Since the uncontrollable and unobservable modes of any system realization are not present in the corresponding system transfer function, $G(z)$, we can truncate the associated states, corresponding to the zeros in $Y$ and $X$ above, and obtain a minimal realization which has both grammians equal to $\Sigma_{1}$. Thus this theorem implies that balanced realizations exist for all stable systems. This is stated as a corollary.

Corollary 1 Every stable system $G(z)$ has a minimal realization which is balanced.

\subsection{Discrete Balanced 1-D Model Reduction}

Using machinery developed for analyzing LFT systems, we present a short proof of the balanced truncation error bound associated with reducing discrete system models. This proof directly generalizes to the MD case, which is given later.

Consider a stable discrete time system $G(z)$ and assume that the transfer matrix has the following realization

$$
G(z)=\left[\begin{array}{c|c}
A & B \\
\hline C & D
\end{array}\right]=\left[\begin{array}{cc|c}
A_{11} & A_{12} & B_{1} \\
A_{21} & A_{22} & B_{2} \\
\hline C_{1} & C_{2} & D
\end{array}\right] \in R \mathcal{H}_{\infty}
$$

Let $P$ and $Q$ be two positive semi-definite symmetric matrices such that

$$
\begin{aligned}
& A P A^{*}-P+B B^{*} \leq 0 \\
& A^{*} Q A-Q+C^{*} C<0 .
\end{aligned}
$$

Note that we have replaced the equalities in (6) and (7) with inequalities. The significance of this is that while the zero-valued eigenvalues of $P$ or $Q$ still have corresponding uncontrollable and/or unobservable states, the converse need not be true. Thus we can truncate states as suggested by Corollary 1 so that $P$ and $Q$ are balanced, exactly as before, but the resulting system may not be minimal. Still, we can assume without loss of generality that

$$
P=Q=\left[\begin{array}{cc}
\Sigma_{1} & 0 \\
0 & \Sigma_{2}
\end{array}\right]
$$

with

$$
\begin{aligned}
& \Sigma_{1}=\operatorname{diag}\left(\sigma_{1} I_{s_{1}}, \sigma_{2} I_{s_{2}}, \ldots, \sigma_{r} I_{s_{r}}\right) \geq 0 \\
& \Sigma_{2}=\operatorname{diag}\left(\sigma_{r+1} I_{s_{r+1}}, \sigma_{r+2} I_{s_{r+2}}, \ldots, \sigma_{n} I_{s_{n}}\right) \geq 0
\end{aligned}
$$

where $s_{i}$ denotes the multiplicity of $\sigma_{i}$ and the partition is assumed to be compatible with the partition of the system. (Note that the singular values $\sigma_{i}$ are not necessarily ordered.)

The following results are standard, but the proof given here of the main result, Theorem 3 , is elementary and can be directly generalized to the M-D case. The term balanced here is used in slightly nonstandard way, as this term is usually applied when equations (6) and (7) are equalities. In the equality case, the $\Sigma_{1}$ is unique, whereas in the inequality case it is not. We will use the term balanced in the looser sense that includes the inequality case.

Theorem 2 If $\Sigma_{1}>0$, then the realization for the truncated subsystem

$$
G_{r}=\left[\begin{array}{c|c}
A_{11} & B_{1} \\
\hline C_{1} & \dot{D}
\end{array}\right]
$$

is balanced and stable.

Proof. The system parameters satisfy the following equations

$$
\begin{aligned}
& A_{11} \Sigma_{1} A_{11}^{*}-\Sigma_{1}+A_{12} \Sigma_{2} A_{12}^{*}+B_{1} B_{1}^{*} \leq 0 \\
& A_{11}^{*} \Sigma_{1} A_{11}-\Sigma_{1}+A_{21}^{*} \Sigma_{2} A_{21}+C_{1}^{*} C_{1} \leq 0
\end{aligned}
$$

But these equations imply that

$$
\begin{aligned}
& A_{11} \Sigma_{1} A_{11}^{*}-\Sigma_{1}+B_{1} B_{1}^{*} \leq 0 \\
& A_{11}^{*} \Sigma_{1} A_{11}-\Sigma_{1}+C_{1}^{*} C_{1} \leq 0
\end{aligned}
$$

hold. The theorem then follows immediately.

Theorem 3 Suppose $G_{r}=\quad\left[\begin{array}{c|c}A_{11} & B_{1} \\ \hline C_{1} & D\end{array}\right] . \quad$ Then $\left\|G-G_{r}\right\|_{\infty} \leq 2 \sum_{i=r+1}^{n} \sigma_{i}$.

In particular, $\|G\|_{+\infty} \leq\|D\|+2 \sum_{i=1}^{n} \sigma_{i}$.

The proof of Theorem 3 will be based on three simple lemmas, which will be proven first. Without loss of generality, we shall assume $\sigma_{n}=1$. We will prove that for $\Sigma_{2}=\sigma_{n} I=I$, we have

$$
\left\|G-G_{r}\right\|_{\infty} \leq 2
$$

The theorem then follows by scaling and recursively applying this result, since the reduced system $G_{r}$ is still balanced.

To simplify the formulas, let $\Lambda=\Sigma_{1}^{1 / 2}$. In the following a constant matrix $X$ is said to be contractive or a contraction if $\|X\|=\bar{\sigma}(X) \leq 1$, and strictly contractive if $\|X\|<1$.

The following well-known lemma $([4],[6])$ establishes the relationship between the $\mathcal{H}_{\infty}$ norm of a transfer matrix and its realizations. We will use only the if part, which is proven later in greater generality in Lemma 6. 
Lemma 1 Suppose $G(z) \in \mathcal{R} \mathcal{H}_{\infty}$ is a discrete time transfer natrix, then $\|G(z)\|_{\infty}<1(\leq 1)$ if and only if there is a realization of $G(z)=$ $\left[\begin{array}{c|c}A & B \\ \hline C & D\end{array}\right]$ such that $\left\|\left[\begin{array}{ll}A & B \\ C & D\end{array}\right]\right\|<1(\leq 1)$.

We may rewrite (6) as

$$
\left[\begin{array}{ll}
A & B
\end{array}\right]\left[\begin{array}{cc}
P & 0 \\
0 & I
\end{array}\right]\left[\begin{array}{l}
A^{*} \\
B^{*}
\end{array}\right] \leq P
$$

from which it follows immediately that

$$
\left[P^{-1 / 2} A P^{1 / 2} \quad P^{-1 / 2} B\right]
$$

is contractive. Similarly, (7) implies that

$$
\left[\begin{array}{c}
Q^{1 / 2} A Q^{-1 / 2} \\
C Q^{-1 / 2}
\end{array}\right]
$$

is contractive. The following lemma simply restates this in a form convenient for later use.

Lemma 2 Suppose a realization of the transfer matrix $G$ satisfies $P=$ $\operatorname{diag}\left\{\Lambda^{2}, I\right\}=Q$, then

$$
\left[\begin{array}{ccc}
\Lambda^{-1} A_{12} & \Lambda^{-1} A_{11} \Lambda & \Lambda^{-1} B_{1} \\
A_{22} & A_{21} \Lambda & B_{2}
\end{array}\right]
$$

and

$$
\left[\begin{array}{cc}
A_{21} \Lambda^{-1} & A_{22} \\
\Lambda A_{11} \Lambda^{-1} & \Lambda A_{12} \\
C_{1} \Lambda^{-1} & C_{2}
\end{array}\right]
$$

are contractive.

Proof. Since from (9)

$$
\begin{gathered}
{\left[\begin{array}{cc}
P^{-1 / 2} A P^{1 / 2} & P^{-1 / 2} B
\end{array}\right]} \\
=\left[\begin{array}{ccc}
\Lambda^{-1} A_{12} & \Lambda^{-1} A_{11} \Lambda & \Lambda^{-1} B_{1} \\
A_{22} & A_{21} \Lambda & B_{2}
\end{array}\right]\left[\begin{array}{ccc}
0 & I & 0 \\
I & 0 & 0 \\
0 & 0 & I
\end{array}\right]
\end{gathered}
$$

is a contraction, we have immediately that

$$
\left[\begin{array}{ccc}
\Lambda^{-1} A_{12} & \Lambda^{-1} A_{11} \Lambda & \Lambda^{-1} B_{1} \\
A_{22} & A_{21} \Lambda & B_{2}
\end{array}\right]
$$

is also a contraction. The second claim follows by a similar argument. $\square$

Finally, the following lemma shows that determining the contractiveness of a large system matrix can be simplified by determining if two related, smaller matrices are contractive.

Lemma 3 Suppose $X=\left[\begin{array}{cc}X_{11} & X_{12} \\ Z & X_{22}\end{array}\right]$ and $Y=\left[\begin{array}{cc}Y_{11} & Z \\ Y_{21} & Y_{22}\end{array}\right]$ are contractive (strictly contractive). Then

$$
M \triangleq\left[\begin{array}{ccc}
0 & \frac{1}{\sqrt{2}} X_{11} & X_{12} \\
\frac{1}{\sqrt{2}} Y_{11} & Z & \frac{1}{\sqrt{2}} X_{22} \\
Y_{21} & \frac{1}{\sqrt{2}} Y_{22} & 0
\end{array}\right]
$$

is also contractive (strictly contractive).
Proof. Dilate $M$ to the following matrix

$$
M_{d} \triangleq\left[\begin{array}{ccccc}
0 & \frac{1}{\sqrt{2}} X_{11} & X_{12} & \vdots & \frac{1}{\sqrt{2}} X_{11} \\
\frac{1}{\sqrt{2}} Y_{11} & Z & \frac{1}{\sqrt{2}} X_{22} & \vdots & 0 \\
Y_{21} & \frac{1}{\sqrt{2}} Y_{22} & 0 & \vdots & -\frac{1}{\sqrt{2}} Y_{22} \\
\cdots & \cdots & \cdots & \cdots & \cdots \\
\frac{1}{\sqrt{2}} Y_{11} & 0 & -\frac{1}{\sqrt{2}} X_{22} & \vdots & -Z
\end{array}\right]
$$

Since $X$ and $Y$ are contractive, it is easy to verify that $M_{d}^{*} M_{d} \leq I$, that is, $M_{d}$ is a contraction.

We can now prove the theorem.

Proof of Theorem 3. Note that

$$
G_{r}=\left[\begin{array}{c|c}
A_{11} & B_{1} \\
\hline C_{1} & D
\end{array}\right]=\left[\begin{array}{cc|c}
A_{11} & 0 & B_{1} \\
0 & 0 & 0 \\
\hline C_{1} & 0 & D
\end{array}\right]
$$

Hence

$$
\frac{1}{2}\left(G-G_{r}\right)=\left[\begin{array}{cccc|c}
A_{11} & 0 & 0 & 0 & \frac{1}{2} B_{1} \\
0 & 0 & 0 & 0 & 0 \\
0 & 0 & A_{11} & A_{12} & \frac{1}{2} B_{1} \\
0 & 0 & A_{21} & A_{22} & \frac{1}{2} B_{2} \\
\hline-C_{1} & 0 & C_{1} & C_{2} & 0
\end{array}\right]
$$

Now apply the following similarity transformation

$$
T=\left[\begin{array}{cccc}
-\Lambda & 0 & \Lambda & 0 \\
0 & -I & 0 & I \\
\Lambda^{-1} & 0 & \Lambda^{-1} & 0 \\
0 & I & 0 & I
\end{array}\right] \quad T^{-1}=\frac{1}{2}\left[\begin{array}{cccc}
-\Lambda^{-1} & 0 & \Lambda & 0 \\
0 & -I & 0 & I \\
\Lambda^{-1} & 0 & \Lambda & 0 \\
0 & I & 0 & I
\end{array}\right]
$$

to the realization of $\frac{1}{2}\left(G-G_{r}\right)$ to get

$$
\frac{1}{2}\left(G-G_{r}\right)=\left[\begin{array}{cccc|c}
\Lambda A_{11} \Lambda^{-1} & \frac{1}{2} \Lambda A_{12} & 0 & \frac{1}{2} \Lambda A_{12} & 0 \\
\frac{1}{2} A_{21} \Lambda^{-1} & \frac{1}{2} A_{22} & \frac{1}{2} A_{21} \Lambda & \frac{1}{2} A_{22} & \frac{1}{2} B_{2} \\
0 & \frac{1}{2} \Lambda^{-1} A_{12} & \Lambda^{-1} A_{11} \Lambda & \frac{1}{2} \Lambda^{-1} A_{12} & \Lambda^{-1} B_{1} \\
\frac{1}{2} A_{21} \Lambda^{-1} & \frac{1}{2} A_{22} & \frac{1}{2} A_{21} \Lambda & \frac{1}{2} A_{22} & \frac{1}{2} B_{2} \\
\hline C_{1} \Lambda^{-1} & \frac{1}{2} C_{2} & 0 & \frac{1}{2} C_{2} & 0
\end{array}\right]
$$

It is easy to verify that as a constant matrix the right hand side of the above realization for $\frac{1}{2}\left(G-G_{r}\right)$ can be written as

$$
\left[\begin{array}{cccc}
0 & 0 & I & 0 \\
0 & \frac{1}{\sqrt{2}} I & 0 & 0 \\
I & 0 & 0 & 0 \\
0 & \frac{1}{\sqrt{2}} I & 0 & 0 \\
0 & 0 & 0 & I
\end{array}\right] \hat{M}\left[\begin{array}{ccccc}
I & 0 & 0 & 0 & 0 \\
0 & \frac{1}{\sqrt{2}} I & 0 & \frac{1}{\sqrt{2}} I & 0 \\
0 & 0 & I & 0 & 0 \\
0 & 0 & 0 & 0 & I
\end{array}\right]
$$

where

$$
\hat{M} \triangleq\left[\begin{array}{cccc}
0 & \frac{1}{\sqrt{2}} \Lambda^{-1} A_{12} & \Lambda^{-1} A_{11} \Lambda & \Lambda^{-1} B_{1} \\
\frac{1}{\sqrt{2}} A_{21} \Lambda^{-1} & A_{22} & \frac{1}{\sqrt{2}} A_{21} \Lambda & \frac{1}{\sqrt{2}} B_{2} \\
\Lambda A_{11} \Lambda^{-1} & \frac{1}{\sqrt{2}} \Lambda A_{12} & 0 & 0 \\
C_{1} \Lambda^{-1} & \frac{1}{\sqrt{2}} C_{2} & 0 & 0
\end{array}\right]
$$

According to Lemma 1, the theorem follows if we can show that the realization for $\frac{1}{2}\left(G-G_{r}\right)$ as a constant matrix is a contraction. However this is guarantced if $\hat{M}$ is a contraction since both the right and left hand matrices in (11) are contractive.

Finally the contractiveness of $\hat{M}$ follows from Lemmas 2 and 3 by identifying

$$
Z=A_{22}, X_{11}=\Lambda^{-1} A_{12}, Y_{11}=A_{21} \Lambda^{-1}
$$

and

$$
\begin{gathered}
{\left[\begin{array}{l}
X_{12} \\
X_{22}
\end{array}\right]=\left[\begin{array}{cc}
\Lambda^{-1} A_{11} \Lambda & \Lambda^{-1} B_{1} \\
A_{21} \Lambda & B_{2}
\end{array}\right]} \\
{\left[\begin{array}{ll}
Y_{21} & Y_{22}
\end{array}\right]=\left[\begin{array}{cc}
\Lambda A_{11} \Lambda^{-1} & \Lambda A_{12} \\
C_{1} \Lambda 1^{-1} & C_{2}
\end{array}\right]}
\end{gathered}
$$




\section{Balanced Model Reduction For MD Sys- tems}

We now consider a generalization of the balanced model reduction technique for 1-D systems to more general LFT systems. We will focus on the case where the $\Delta$ block is a diagonal matrix, which may be thought of as representing a multi-dimensional frequency structure. In this case, the LFT systems represent conventional multi-dimensional (MD) systems, and the terms will be used interchangeably. The results here generalize directly to $\Delta$ that include full blocks, but the notation is cumbersome so it will be omitted. Also, the full block version does not have any MD interpretation, although it maintains an interpretation as an uncertain system.

\subsection{Balanced MD Systems}

Consider the system in Figure 1 with the frequency structure $\Delta$ specified from (1) and (2). Let $M=\left[\begin{array}{ll}A & B \\ C & D\end{array}\right]$ be a complex matrix with $A, B$ and $C$ partitioned compatibly with the block structure $\Delta$ as

$$
A=\left[\begin{array}{ccc}
A_{11} & \cdots & A_{1 r} \\
\vdots & \ddots & \vdots \\
A_{r 1} & \cdots & A_{r r}
\end{array}\right] ; \quad B=\left[\begin{array}{c}
B_{1} \\
\vdots \\
B_{r}
\end{array}\right] ; \quad C=\left[\begin{array}{lll}
C_{1} & \cdots & C_{r}
\end{array}\right] .
$$

The input/output mapping for this MD system is given by

$$
y=\mathcal{F}_{u}(M, \Delta) u
$$

where

$$
\mathcal{F}_{\mathrm{u}}(M, \Delta)=D+C \Delta(I-A \Delta)^{-1} B .
$$

For MD systems, the concept of stability referred to in the following discussion of model reduction is that of $\mathcal{Q}$-stability, which corresponds to the SSUB in [6].

Definition 1 The $M D$ system $\mathcal{F}_{u}(M, \Delta)$ is said to be $Q$-stable if there exists a non-singular matrix $T$ such that $T \Delta=\Delta T \forall \Delta$ and

$$
\overline{\boldsymbol{\sigma}}\left(T A T^{-1}\right)<1 .
$$

Since $\Delta=\operatorname{diag}\left[\delta_{1} I_{q_{1}}, \cdots, \delta_{r} I_{q r}\right]$ where $\delta_{i} \in \mathrm{C}$, to satisfy the commutative condition $T \Delta=\Delta T, T$ must have the structure of $T=\operatorname{diag}\left[T_{1}, \cdots, T_{r}\right]$. As in [6], we can express $\mathcal{Q}$-stability in an LMI form. Define positive definite matrices $P$ and $Q$ by $P=\left(T T^{* *}\right)^{-1}$ and $Q=T^{*} T$. Then $P$ and $Q$ have the structures of $P=\operatorname{diag}\left[P_{1}, \cdots, P_{r}\right]$ and $Q=\operatorname{diag}\left[Q_{1}, \cdots, Q_{r}\right]$. Recall from [6] that

$$
\begin{aligned}
& \bar{\sigma}\left(T A T^{-1}\right)<1 \\
\Longleftrightarrow & T A T^{-1}\left(T^{-1}\right)^{*} A^{*} T^{*}-I<0 \\
\Longleftrightarrow & A P A^{*}-P<0 \\
\Longleftrightarrow & A^{*} Q A-Q<0
\end{aligned}
$$

From (13) and scaling of $P$ and $Q$ one can immediately deduce the following lemma.

Lemma 4 The $M D$ system $\mathcal{F}_{u}(M, \Delta)$ is $\mathcal{Q}$-stable if and only if there exist $P>0$ and $Q>0$ such that the following inequalities hold.

$$
\begin{aligned}
& A P A^{*}-P+B B^{*}<0 \\
& A^{*} Q A-Q+C^{*} C<0
\end{aligned}
$$

Remark 1 We can also mimic the one-dimensional case by using nonstrict inequalities. Then it is easy to see that if $\mathcal{F}_{u}(M, \Delta)$ is $\mathcal{Q}$-stable, there exist $P \geq 0$ and $Q \geq 0$ such that the following inequalities hold.

$$
\begin{aligned}
& A P A^{*}-P+B B^{*} \leq 0 \\
& A^{*} Q A-Q+C^{*} C \leq 0
\end{aligned}
$$

Although these inequalities can be used to derive the model reduction error bound, the condition for the reduced model stability is messy. Thus for simplicity of exposition, we will consider only the case of strict inequality, as in Lemma 4 .
As in the 1-D case, a similarity transformation for a MD system is an invertible matrix, $T$, which transforms the system states. In the following lemma, a general formula for a similarity transformation on a LFT system is presented. The proof follows from a straightforward application of the definition of $\mathcal{F}_{u}(M, \Delta)$.

Lemma 5 For any nonsingular complex matrix $T$

$$
\mathcal{F}_{u}(M, \Delta)=\mathcal{F}_{u}\left(\left[\begin{array}{cc}
T A T^{-1} & T B \\
C T^{-1} & D
\end{array}\right], T \Delta T^{-1}\right)
$$

In particular, if the non-singular matrix $T$ has the structure $T=$ $\operatorname{diag}\left[T_{1}, \cdots, T_{r}\right]$ so that $T$ and $\Delta$ commute, then

$$
\mathcal{F}_{u}(M, \Delta)=\mathcal{F}_{u}\left(\left[\begin{array}{cc}
T A T^{-1} & T B \\
C T^{-1} & D
\end{array}\right], \Delta\right) .
$$

Note that the structured similarity transformation $T=$ $\operatorname{diag}\left[T_{1}, \cdots, T_{r}\right]$ produces the same effect on the $P$ and $Q$ which are the solutions of the inequalities (14) and (15), as in the 1-D case. If we refer to the different $M$ produced by such similarity transformations as different realizations, just as in the $1 \mathrm{D}$ case, then there exists MD balanced realizations, just as in the $1 \mathrm{D}$ case.

Deflnition 2 If a MD system satisfies inequalities (14) and (15) and

$$
P=Q=\Sigma=\operatorname{diag}\left[\Sigma_{1}, \cdots, \Sigma_{r}\right]
$$

where $\Sigma_{i}=\operatorname{diag}\left[\sigma_{i 1} I_{s_{i 1}}, \cdots, \sigma_{i t_{i}} I_{s_{1, i}}\right]>0 ; \sigma_{i 1} \geq \cdots \geq \sigma_{i t_{i}}$ and the dimension of block $\Sigma_{i}$ is $q_{i}=\sum_{j=1}^{t_{i}} s_{i j}$, then the system is said to be balanced. The matrix $\Sigma$ is called a structured gramian of the $M D$ system and every $\Sigma_{i}, i=1, \cdots, r$ is called a block gramian.

Thus Lemma 5 guarantees the existence of balanced realizations, which we state as a corollary. Note that neither the balanced $P$ and $Q$ nor the resulting realization is unique.

Corollary 2 If $\mathcal{F}_{u}(M, \Delta)$ is $\mathcal{Q}$-stable, then there exists a realization of $\mathcal{F}_{u}(M, \Delta)$ such that $P=Q=\Sigma=\operatorname{diag}\left[\Sigma_{1}, \cdots, \Sigma_{r}\right]>0$, where $P$ and $Q$ are the solution of the inequalities (14) and (15) respectively.

\subsection{Error Bound for MD Model Reduction}

For a balanced MD system, let every block gramian be partitioned as

$$
\Sigma_{i}=\operatorname{diag}\left[\hat{\Sigma}_{1 i}, \Sigma_{2 i}\right]
$$

for $i=1, \cdots, r$, where

$$
\hat{\Sigma}_{1 i}=\operatorname{diag}\left[\sigma_{i 1} I_{s_{i 1}}, \cdots, \sigma_{i k_{i}} I_{s_{i k_{i}}}\right], \quad k_{i} \leq t_{i}
$$

and

$$
\Sigma_{2 i}=\operatorname{diag}\left[\sigma_{i\left(k_{i}+1\right)} I_{s_{i\left(k_{i}+1\right)}}, \cdots, \sigma_{i t_{i}} I_{s, t_{i}}\right] .
$$

Truncate both $\Sigma_{2 i}$ and the corresponding parameter matrices

$$
\begin{aligned}
& M=\left[\begin{array}{cc}
A & B \\
C & D
\end{array}\right]=\left[\begin{array}{cccc}
A_{11} & \cdots & A_{1 r} & B_{1} \\
\vdots & \ddots & \vdots & \vdots \\
A_{r 1} & \cdots & A_{r r} & B_{r} \\
C_{1} & \cdots & C_{r} & D
\end{array}\right] \\
& =\left[\begin{array}{cccccc}
\hat{A}_{11} & A_{11_{12}} & \cdots & \hat{A}_{1 r} & A_{1 r_{12}} & \hat{B}_{1} \\
A_{11_{21}} & A_{11_{22}} & \cdots & A_{1 r_{21}} & A_{1 r_{22}} & B_{12} \\
\vdots & \vdots & \ddots & \vdots & \vdots & \vdots \\
\hat{A}_{r 1} & A_{r 1_{12}} & \cdots & \hat{A}_{r r} & A_{r r_{2}} & \hat{B}_{r} \\
A_{r 1_{21}} & A_{r_{22}} & \cdots & A_{r r_{21}} & A_{r r_{22}} & B_{r_{2}} \\
\hat{C}_{1} & C_{1_{2}} & \cdots & \hat{C}_{r} & C_{r_{2}} & D
\end{array}\right]
\end{aligned}
$$

such that the reduced system after being truncated is

$$
\hat{M}=\left[\begin{array}{cc}
\hat{A} & \hat{B} \\
\hat{C} & D
\end{array}\right]=\left[\begin{array}{cccc}
\hat{A}_{11} & \cdots & \hat{A}_{1 r} & \hat{B}_{1} \\
\vdots & \ddots & \vdots & \vdots \\
\hat{A}_{r 1} & \cdots & \hat{A}_{r r} & \hat{B}_{r} \\
\hat{C}_{1} & \cdots & \hat{C}_{r} & D
\end{array}\right] .
$$


with frequency structure $\hat{\Delta}=\operatorname{diag}\left[\delta_{1} I_{\hat{q}_{1}}, \ldots, \delta_{r} I_{\hat{q}_{r}}\right]$ where $\hat{q}_{i}=$ $\sum_{j=1}^{k_{i}} s_{i j}$. This model reduction method will be called bulanced $M D$ model reduction.

The following lemma is a direct generalization of one direction of Lemma 1

Lemma 6 Suppose $\mathcal{F}_{u}(M, \Delta)$ is $Q$-stable for a given frequency structure $\Delta$. If there exists a realization $M$ such that $\|M\| \leq 1(<1)$, then $\left\|\mathcal{F}_{u}(M, \Delta)\right\| \leq 1(<1)$ for all $\|\Delta\| \leq 1$.

Proof. By definition

$$
\left[\begin{array}{c}
y_{1} \\
y
\end{array}\right]=M\left[\begin{array}{c}
u_{1} \\
u
\end{array}\right], \quad u_{1}=\Delta y_{1}
$$

Hence if $\|M\| \leq 1(<1)$ and $\|\Delta\| \leq 1$, we have

$$
\left\|u_{1}\right\| \leq\left\|y_{1}\right\|
$$

and

$$
\begin{aligned}
\|y\|^{2}+\left\|y_{1}\right\|^{2} & \leq\|M\|^{2}\left(\|u\|^{2}+\left\|u_{1}\right\|^{2}\right) \\
& \leq\|u\|^{2}+\left\|u_{1}\right\|^{2} \quad\left(<\|u\|^{2}+\left\|u_{1}\right\|^{2}\right) \\
& \leq\|u\|^{2}+\left\|y_{1}\right\|^{2}\left(<\|u\|^{2}+\left\|y_{1}\right\|^{2}\right)
\end{aligned}
$$

that is,

$$
\|y\|^{2} \leq\|u\|^{2}\left(<\|u\|^{2}\right)
$$

and since this holds for all $u$, we have that

$$
\left\|\mathcal{F}_{\mathbf{u}}(M, \Delta)\right\| \leq 1(<1)
$$

It is easily verified that, exactly as in the $1 \mathrm{D}$ case, the truncation of a balanced $\mathcal{Q}$-stable MD system is still balanced and $\mathcal{Q}$-stable. We now state and prove the main result of this paper. The notation $\left\|\mathcal{F}_{u}(M, \Delta)\right\|_{\infty}$ means maximum over all $\Delta \in \mathbf{B} \Delta$, and where relevant, we assume that the corresponding parts of $\Delta$ and $\hat{\Delta}$ are equal.

Theorem 4 Suppose $\mathcal{F}_{u}(\hat{M}, \hat{\Delta})$ is the reduced balanced model obtained from $\mathcal{F}_{u}(M, \Delta)$. Then $\mathcal{F}_{u}(\hat{M}, \hat{\Delta})$ is $\mathcal{Q}$-stable and

$$
\left\|\mathcal{F}_{u}(\boldsymbol{M}, \Delta)-\mathcal{F}_{u}(\hat{M}, \hat{\Delta})\right\|_{\infty} \leq 2 \sum_{i=1}^{r} \sum_{j=k_{1}+1}^{t_{i}} \sigma_{i j}
$$

In particular, $\left\|\mathcal{F}_{u}(M, \Delta)\right\|_{\infty} \leq\|D\|+2 \sum_{i=1}^{r} \sum_{j=1}^{t_{i}} \sigma_{i j}$.

Proof. We will prove this theorem recursively, just as in the 1-D case. By scaling and permutation of the $\delta_{i}$, if necessary, we can assume without loss of generality that the system is reduced from

$$
M=\left[\begin{array}{ccc}
\hat{A} & A_{12} & \hat{B} \\
A_{21} & A_{22} & B_{2} \\
\hat{C} & C_{2} & D
\end{array}\right]
$$

to $\hat{M}=\left[\begin{array}{cc}\hat{A} & \hat{B} \\ \hat{C} & D\end{array}\right]$, with $\Sigma=\operatorname{diag}[\hat{\Sigma}, I]$. The corresponding frequency structures are reduced from $\Delta=\operatorname{diag}\left[\delta_{1} I_{q_{1}}, \cdots, \delta_{r} I_{q_{r}}\right]$ to $\hat{\Delta}=\operatorname{diag}\left[\delta_{1} I_{q_{1}}, \cdots, \delta_{\mathrm{r}} I_{\hat{q}_{r}}\right]$, in which $\hat{q}_{r}=\sum_{j=1}^{\left(t_{r}-1\right)} s_{r j}<q_{r}$, that is, only the states corresponding to the last frequency variable $\delta_{r}$ in $\Delta$ and the last singular value $\sigma_{r t_{r}}=1$ in $\Sigma_{r}$ is reduced. The theorem reduces to this case by repeated scaling, rearrangement, and truncation.

The remainder of the proof is nearly identical to the corresponding proof for the 1-D case. As in the example preceeding Theorem 4, the reduced system $\mathcal{F}_{u}(\hat{M}, \hat{\Delta})$ is still balanced and satisfies the strict inequalities. So the balanced system is $\mathcal{Q}$-stable. For convenience, we set $\Lambda=\hat{\Sigma}^{1 / 2}$ in the following proof. Let

$$
\mathcal{F}_{u}\left(M_{g}, \Delta_{g}\right)=\frac{1}{2}\left(\mathcal{F}_{u}(M, \Delta)-\mathcal{F}_{u}(\hat{M}, \hat{\Delta})\right)
$$

be the error system with

$$
M_{g}=\left[\begin{array}{ccccc}
\dot{A} & 0 & 0 & 0 & \frac{1}{2} \hat{B} \\
0 & 0 & 0 & 0 & 0 \\
0 & 0 & \hat{A} & A_{12} & \frac{1}{2} \hat{B} \\
0 & 0 & A_{21} & A_{22} & \frac{1}{2} B_{2} \\
-\hat{C} & 0 & \hat{C} & C_{2} & 0
\end{array}\right]
$$

and $\Delta_{g}=\operatorname{diag}[\Delta, \Delta]$. One can easily prove that $\Delta_{g}$ in $\mathcal{F}_{u}\left(M_{g}, \Delta_{g}\right)$ will remain unchanged by the similarity transformation $T$ in (10). Applying this similarity transformation to the system represented by $\boldsymbol{M}_{g}$, one obtains the following system matrix, denoted by $\tilde{M}_{g}$,

$$
\dot{M}_{g}=\left[\begin{array}{ccccc}
\Lambda \hat{A} \Lambda^{-1} & \frac{1}{2} \Lambda A_{12} & 0 & \frac{1}{2} \Lambda A_{12} & 0 \\
\frac{1}{2} A_{21} \Lambda^{-1} & \frac{1}{2} A_{22} & \frac{1}{2} A_{21} \Lambda & \frac{1}{2} A_{22} & \frac{1}{2} B_{2} \\
0 & \frac{1}{2} \Lambda^{-1} A_{12} & \Lambda^{-1} \hat{A} \Lambda & \frac{1}{2} \Lambda^{-1} A_{12} & \Lambda^{-1} \hat{B} \\
\frac{1}{2} A_{21} \Lambda^{-1} & \frac{1}{2} A_{22} & \frac{1}{2} A_{21} \Lambda & \frac{1}{2} A_{22} & \frac{1}{2} B_{2} \\
\hat{C} \Lambda^{-1} & \frac{1}{2} C_{2} & 0 & \frac{1}{2} C_{2} & 0
\end{array}\right] .
$$

By Lemma 6 , if $\dot{M}_{g}$ is contractive, then $\left\|\mathcal{F}_{u}(M, \Delta)-\mathcal{F}_{u}(\hat{M}, \hat{\Delta})\right\|=$ $2\left\|\mathcal{F}_{u}\left(M_{g}, \Delta_{g}\right)\right\|_{\infty} \leq 2$. But Lemma 2 and 3 can be used exactly as in the 1-D case in Theorem 3 to show that $\tilde{M}_{g}$ is a contraction. By applying this procedure recursively, the theorem follows.

\subsection{Interpretations}

It is easy to see that the strict inequality holds in inequality (19). It is also easy to check that all results hold if the $P$ and $Q$ inequalities (16) and (17) are used except that we can not easily conclude that the reduced model is $\mathcal{Q}$-stable.

Note that what is shown in the proof of Theorem 4 is actually much stronger than (19). Using the terminology of [6], we have proven that the error system in (20) and (21) satjsfies the SSUB (has $\mathcal{Q}$ robust performance), whereas (19) would be implied by the weaker $\mu$ robust performance. One implication of this is that the error system bounded in (19) holds for the $l_{2}$ induced norm for arbitrary time-varying and/or nonlinear $l_{2}$ induced norm bounded perturbations. This is typical for generalizations of results for standard transfer functions to general LFTs; it is easiest to use the $\mathcal{Q}$ theory.

In the 1- $d$ case there are lower bounds in addition to the upper bound in (19), but these have not yet been generalized to the MD case.

\section{References}

[1] Agathoklis, P., 1988, The Lyapunov equation for $n$-dimensional discrete systems, IEE'E Trans. on Circuits ans Systems, Vol. CAS35:448-451.

[2] Anderson, B.D.O., Agathoklis, P., Jury, E.I. and Mansour, M., 1986, Stability and the matrix Lyapunov equation for discrete 2dimensional systems, IEEE Trans, on Circuits ans Systems, Vol. CAS-33:261-267.

[3] Anderson, B.D.O. and Moore, G., 1979, Optimal Filtering.

[4] Boyd, S. and Yang, Q., 1988, Simultaneous Quadratic Lyapunov Functions, Stanford Research Report.

[5] Desai, U.B. and Pal, D., 1984, A transformation approach to stochastic model reduction, IEEE Trans. AC-29:1097-1100.

[6] Doyle, J., Packard, A., and Zhou, K., LFTs, LMIs, and $\mu, 1991$ CDC, Brighton, UK.

[7] Enns, D.F., 1984, Model Reduction for Control System Design, Ph.D. Dissertation, Stanford University.

[8] Glover, K., 1984, All optimal Hankel-norm approximations of linear multivariable systems and their $L^{\infty}$-error bounds, Int. J. Control., Vol.39:1115-1193.

[9] Hinrichsen, D. and Pritchard, A.J., 1990, An improved error estimate for reduced-order models of discrete-time system, IEEE Trans, on Auto. Control, AC-35:317-320. 
[10] IEEE Proceeding, 1977, Special issue on multidimensional systems.

[1I] Lu, W. and Lee, E.B., 1985, Stability analysis for two-dimensiona] systems via a Lyapunov approach, IEEE Trans. on Circuits ans Systems, Vol. CAS-32:61-68.

[12] B.C. Moore, Principal component analysis inear systems: Controllability, observability and model reduction, IEEE Trans. Automat. Control, 26 (1981) 17-32.

[13] Packard, A. and Doyle, J., 1990, Quadratic stability with real and complex perturbations, IEEE Trans. on Auto. Control, AC35:198-201.

[14] Premaratne, K., Jury, E.I. and Mansour, M., 1990, An algorithm for model reduction of 2-D discrete time systems, IEEE Trans. on Circuits and Systems, Vol:CAS-37:1116-1132.

[15] Wang, W. and Safonov, M.G., 1990, A relative error bound for discrete-time balanced stochastic truncation, Proc. American Control Conf., (San Diego, CA, May 23-25, 1990); also expended version to be appeared in Int. J. of Control.

[16] Wang, W., 1990, Relative-Error Model Reduction, Identification, and Control, Ph.D. Dissertation, The University of Southern California.

[17] Zhou, K. and Doyle, J., 1990, Notes on MIMO Control Theory, unpublished note 